\title{
AGGREGATE LAND RENTS AND AGGREGATE TRANSPORT GOSTS*
}

This paper explores the relationship between aggregate land rents and aggregate transport costs for land markets in which locations differ solely in terms of accessibility. ${ }^{1}$

That there exists a relationship between land rents and transport costs has been recognised at least since the time of von Thünen. ${ }^{2}$ The precise relationship between the two is, however, not generally well-understood. For instance, until quite recently it was considered correct to estimate the benefits from a transport improvement by the induced change in aggregate land rents at those locations where travel costs are reduced. This procedure can be shown to be correct only in very special circumstances. ${ }^{3}$ This paper presents a very general characterisation of the relationship between aggregate land rents and aggregate transport costs. In some special cases, the relationship turns out to be remarkably simple: for a circular city with linear transport costs, aggregate transport costs are precisely twice aggregate land rents, independent of the distribution of tastes or income; ${ }^{4}$ for a linear city with linear transport costs, aggregate transport costs are equal to aggregate land rents. One corollary of our general analysis is that aggregate

* This paper draws on Arnott's Ph.D. thesis (Arnott, 1975). He would like to thank the Canada Council for financial support during the period the thesis was being written. Stiglitz would like to thank the National Science Foundation for financial support. The comments of Ronald Grieson and two anonymous referees were helpful. The research reported here is part of the NBER's research programme in taxation. Any opinions expressed are those of the authors and not those of the National Bureau of Economic Research.

1 Thus, we abstract from differences in the site-intrinsic or Ricardian characteristics of land.

2 In the von Thünen model differences in agricultural land rents are related to the costs of transporting goods to the central market.

3 The argument underlying this procedure is based on partial equilibrium analysis. If transport costs to all locations but one small location were to remain unchanged, then the benefits from the transport improvement would be correctly measured by the induced change in land rents at that location. But as in most instances in spatial economics, partial equilibrium analysis is inappropriate, and general equilibrium analysis should be applied. The incorrectness of this procedure was persuasively argued in an important paper by Mohring (1961) twenty years ago; one still, however, comes across analyses that use some variant of it (Fishlow (1965), Fogel (1964) for instance). The conditions under which the above partial equilibrium argument is appropriate can be seen by considering a resident's indirect utility function. In a spatial economy, a resident's utility is a function of the land rent where he locates, $R$, his income net of transport costs, $I$, and other prices, $p$, so that $V=V(R, I, p)$, where $V$ is the indirect utility function. Where other prices are unaffected by the transport improvement, $d V=V_{1} d R+V_{2} d I$. From the properties of the indirect utility function $V_{1}=-T V_{2}$, where $T$ is the resident's lot size. Thus,

$$
d V=V_{2}(-T d R+d I),
$$

from which it follows that the benefits to this resident from the transport improvement, $d I$, equal the change in his land rents, $T d R$, only when $d V=0$. Thus, only when the transport improvement leaves unchanged the utility of residents at locations to which travel costs have been reduced (which will occur when the city is completely open, for instance) is the partial equilibrium analysis appropriate. This line of argument is developed further in Polinsky and Shavell (1976).

4 Mohring (196r) calculated the relationship between aggregate transport costs and aggregate land rents in a circular city with linear transport costs for the rather special case where all individuals have identical and fixed lot sizes. His method of analysis, summarised below in footnote 1, p. 333, does not, however, generalise to other cases. 
land rents may stay the same or actually fall in response to a transport improvement which makes everyone better off. ${ }^{1}$

In the first section we consider a simple example. The second derives the basic theorems of the paper, while the third examines their implications for the relationship between the benefits from a transport improvement and the change in aggregate land rents induced by the improvement. And in the fourth section, we examine the extent to which the theorems of section II generalise.

\section{A SIMPLE EXAMPLE}

We employ the standard residential location model but, as we shall note later, many of our results extend to more general models. In this model there is a single city centre, a point in space, at which all non-residential activity takes place. Land is used only for the housing of identical city residents who live at different distances from the city centre. If cities do not border on one another, if transport costs are simply a function of the crow-line distance from the city centre, and if land is homogeneous, then the city is circular. If, however, the whole plain is occupied by cities, then, under the above conditions, every city is hexagonal. ${ }^{2}$ Later we consider cities with other geographical configurations and cities in which transport costs are not simply a function of crow-line distance from the city centre.

The identical individuals derive utility from lot size and private goods, and have no preference for location per se. Trip frequency is fixed, and all transport costs are money costs. ${ }^{3}$ In this particular example, we assume that everyone lives on a lot of unit size. ${ }^{4}$ Since land is homogeneous, differences in land rents reflect only differences in transport costs. Specifically, if $R(t)$ is the rent per unit area of land at distance $t$ from the city centre, and $f(t)$ are transport costs to the location, then

$$
R^{\prime}(t)=-f^{\prime}(t)
$$

where the prime indicates the derivative with respect to $t$. For the circular city, with the boundary $t^{*}$ from the centre, aggregate land rents $(A L R)$ equal

$$
A L R \equiv \int_{0}^{t^{*}} R(t) 2 \pi t d t .
$$

Aggregate land rents are calculated as the rent per unit area of land at a distance $t$ from the centre times the number of units of land between $t$ and $t+d t(2 \pi t d t)$, integrated over all $t$.

1 The same result, for a somewhat different type of urban model, has been derived by Getz (1975).

2 This result can be obtained easily for identical individuals in identical cities by application of theorems presented in Bollobas and Stern (1972).

${ }^{3}$ In Section IV, the analysis is extended to situations in which there are time as well as money costs involved in travel. In this case aggregate transport costs include the monetised value of the time spent in travel. Variable trip frequency is treated in footnote 2, p. 339.

4 This would occur under competition if the utility function were of the form $U=\left\{\begin{array}{l}0 \text { if } T<\mathrm{I} \\ C \text { if } T \geqslant \mathrm{I}\end{array}\right.$, where $T$ is lot size and $C$ other goods. 
Similarly, aggregate transport costs $(A T C)$ equal

$$
A T C \equiv \int_{0}^{t^{*}} f(t) 2 \pi t d t .
$$

Integrating (2) by parts, and substituting (I), we obtain

$$
A L R=\int_{0}^{t^{*}}-R^{\prime} \pi t^{2} d t+R\left(t^{*}\right) \pi t^{* 2}=\int_{0}^{t^{*}} f^{\prime} \pi t^{2} d t+R\left(t^{*}\right) \pi t^{* 2} .
$$

The second term on the right-hand side is just the area of the city times the rent on marginal land; hence, the first term is differential land rents. Denoting differential land rents by $D L R$, we observe that

$$
D L R \gtreqless \frac{1}{2} A T C \text { when } \frac{f^{\prime} t}{f} \gtreqless \mathrm{I} \text { for all } t .
$$

$f^{\prime} t / f$ is the elasticity of the transport cost function. In the special case of linear transport costs, differential land rents are precisely one-half aggregate transport costs. $^{1}$

\section{BASIC THEOREMS}

This section generalises the example of the previous section in two ways. First, we allow for arbitrary tastes, and second we treat cities with arbitrary geographical configurations. Residents are still identical.

Let $T(t)$ be the amount of land the individual at $t$ resides on, $R(t)$ be the rental price per unit area of land at $t$, and $I(t)$ be income net of transport costs at $t$. The indirect utility function of the individual at $t$ is $V(R(t), I(t))$. Note that since land is homogeneous and individuals have no preference for location per se, $t$ does not enter $V()$ as a separate argument. Maximisation of utility with respect to $t$ gives

$$
V_{1}(t) R^{\prime}(t)=V_{2}(t) f^{\prime}(t)
$$

and since $-V_{2}(t) T(t)=V_{1}(t)$, then

$$
R^{\prime}(t)=\frac{-f^{\prime}(t)}{T(t)}
$$

We characterise the shape of the city by the function $\Phi(t)$, which gives the residential area within a travelling distance $t$ from the centre of the city. Thus, $\Phi^{\prime}(t) d t$ gives the residential land area between distances $t$ and $t+d t$ from the city centre. There are many geographical configurations for cities that have the same

1 Mohring ( $196 \mathrm{r}$ ) analysed this case of a circular city with linear transport costs and uniform lot size. He provided an ingenious geometric explanation of this result. From ( 1 ) each person's expenditure on land rent plus transport costs is the same. Since each person resides on a lot of unit size, land rent plus transport costs are constant per unit area, $k^{\prime \prime}$, over the settled area of the city. Consider plotting land rent plus transport costs per unit area on the $z$-axis, where the $x$ - $y$ plane is the homogeneous plain on which the city is located. The graphed figure is a cylinder with radius $t^{*}$ and height $k^{\prime \prime}$. The volume of the cylinder is aggregate transport costs plus aggregate land rents. Aggregate land rents are given by the volume of a cone with the same base and height. Since the volume of a cone is one-third that of a cylinder with the same base and height, aggregate land rents are one-half aggregate transport costs. Unfortunately, this neat geometric interpretation does not extend easily to situations in which lot size varies with location. 
shape according to our definition. This is illustrated in Fig. I. The amount of residential land area within a travelling distance $t$ of the city centre is the same in both cities for all $t$, even though their geographical configurations are quite different.
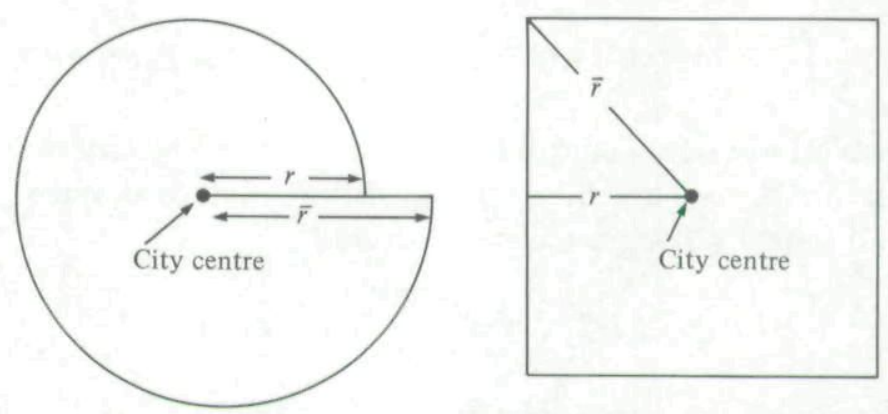

Fig. 1. Two cities with different geographical configurations but the same shape.

Proceeding as in the example, we have

$$
\begin{aligned}
A L R & =\int_{0}^{t^{*}} R(t) \Phi^{\prime}(t) d t \\
& =\int_{0}^{t^{*}}-R^{\prime}(t) \Phi(t) d t+R\left(t^{*}\right) \Phi\left(t^{*}\right),
\end{aligned}
$$

where $t^{*}$ is the furthest distance of settlement from the city centre. Combining (7) and (8) gives

$$
D L R=\int_{0}^{t^{*}} \frac{\mathrm{I}}{T(t)}\left[f^{\prime}(t) \Phi(t)\right] d t
$$

Aggregate transport costs are

$$
A T C=\int_{0}^{t^{*}} \frac{\mathrm{I}}{T(t)}\left[f(t) \Phi^{\prime}(t)\right] d t .
$$

Thus, from (9) and (10),

$$
\frac{A T C}{D L R}=\frac{\int_{0}^{t^{*}} \frac{\mathrm{I}}{T(t)}\left[f(t) \Phi^{\prime}(t)\right] d t}{\int_{0}^{t^{*}} \frac{\mathrm{I}}{T(t)}\left[f^{\prime}(t) \Phi(t)\right] d t},
$$

which is the central result of the paper. Equation (II) indicates that the ratio of aggregate transport costs to differential land rents depends critically on $\left(f \Phi^{\prime}\right) /\left(f^{\prime} \Phi\right)$. Since $\left(f \Phi^{\prime}\right) /\left(f^{\prime} \Phi\right)=\left[f /\left(f^{\prime} t\right)\right]\left[\left(\Phi^{\prime} t\right) / \Phi\right]$, this term equals the elasticity of the shape of the city with respect to distance from the city centre divided by the elasticity of transport costs with respect to distance from the city centre. Some implications of (II) are given in the following Theorem and Corollaries. 
Theorem 1: If $\frac{f \Phi^{\prime}}{f^{\prime} \Phi} \gtreqless \delta$ (a constant) for all $t$, then $A T C \gtreqless \delta D L R$.

Corollary 1 : If $\frac{\Phi^{\prime} t}{\Phi}=\beta$ (a constant) for all $t$, then

$$
A T C \geqq \frac{\beta}{\gamma} D L R \text { as } \frac{f^{\prime} t}{f} \leqq \gamma \text { for all } t \text {. }
$$

Corollary 2: If $\frac{f^{\prime} t}{f}=\gamma$ (a constant) for all $t$, then

$$
A T C \gtreqless \frac{\beta}{\gamma} D L R \quad \text { as } \quad \frac{\Phi^{\prime} t}{\Phi} \gtreqless \beta \text { for all } t \text {. }
$$

We now apply the Theorem and its Corollaries to a number of special cases.

(a) If there are no natural obstacles and if cities do not border on one another, the city is circular, in which case $\Phi=\pi t^{2}$ and $\Phi^{\prime} t / \Phi=2$. Thus, from Corollary $\mathrm{I}$,

$$
A T C \gtreqless \frac{2}{\gamma} D L R \text { as } \frac{f^{\prime} t}{f} \lesseqgtr \gamma \text { for all } t .
$$

If, for instance, there are fixed costs and constant marginal costs associated with travel, then $A T C>2 D L R$ since $f^{\prime} t / f<\mathrm{I}$. And in a circular city with linear transport costs, aggregate transport costs are precisely twice differential land rents.

(b) In a linear city of width $\bar{w}, \Phi=\bar{w} t$ and $\Phi^{\prime} t / \Phi=\mathrm{I}$, and

$$
A T C \gtreqless \frac{\mathrm{I}}{\gamma} D L R \text { as } \frac{f^{\prime} t}{f} \leqq \gamma \text { for all } t \text {. }
$$

With linear transport costs, differential land rents equal aggregate transport costs.

(c) In a hexagonal city,

$\Phi=\left\{\begin{array}{l}\pi t^{2} \text { for } t \leqslant(\sqrt{ } 3 / 2) t^{*} \\ 6\left\{(\sqrt{ } 3 / 2) t^{*} \sqrt{ }\left(t^{2}-\frac{3 t^{* 2}}{4}\right)+\left[\frac{\pi}{6}-\cos ^{-1}\left(\frac{\sqrt{ } t^{*}}{2 t}\right)\right] t^{2}\right\} \text { for } t^{*} \geqslant t \geqslant(\sqrt{3} / 2) t^{*},\end{array}\right.$

where $t^{*}$ is the outer radius of the hexagon. For such a city, $\Phi^{\prime} t / \Phi \leqslant 2$ for all $t$ with strict inequality for some $t$, so that

$$
A T C<\frac{2}{\gamma} D L R \quad \text { if } \quad \frac{f^{\prime} t}{f} \geqq \gamma \quad \text { for all } t .
$$

We define a city to be $C$-concave if $\Phi^{\prime \prime} t<\Phi^{\prime}$ for all $t$. Such cities have the property that, if the city centre is a point, there is a city of the same shape for which a straight line joining any location in the city to the centre lies entirely inside the city. Similarly, a $C$-convex city is one for which $\Phi^{\prime \prime} t>\Phi^{\prime}$ for all $t$. Fig. 2 shows three city shapes, one $C$-concave, another $C$-convex, and another which is neither. 
(d) In a $C$-concave city, ${ }^{1}$

$$
A T C<\frac{2}{\gamma} D L R \text { if } \frac{f^{\prime} t}{f} \geqslant \gamma \text { for all } t .
$$

(e) In a $C$-convex city,

$$
A T C>\frac{2}{\gamma} D L R \text { if } \frac{f^{\prime} t}{f} \leqslant \gamma \text { for all } t .
$$

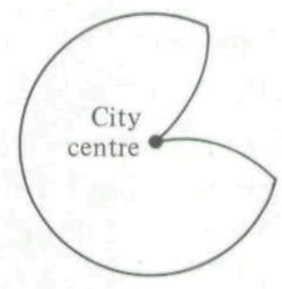

(i) A C-concave city

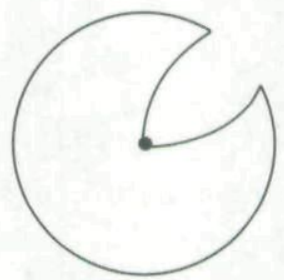

(ii) $\mathrm{AC}$-convex city

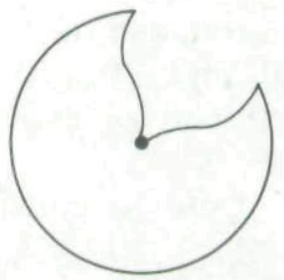

(iii) A city that is neither $C$-convex nor $C$-concave

Fig. 2. Alternative city shapes.

In our analysis, what is relevant is the area which can be reached with a given expenditure in transport costs. We may thus use the cost of transport, $f$, as our measure of distance, and may define the transport cost shape of the city, denoted by $\Omega(f)$, to be the residential land area for which travel costs are less than or equal to $f$. Now, $\Omega[f(t)]=\Phi(t)$. Letting $\Omega^{\prime}$ denote $d \Omega / d f$, then $\Omega^{\prime} f^{\prime}=\Phi^{\prime}$. Combining these two results gives

$$
\frac{\Omega^{\prime} f}{\Omega}=\frac{\Phi^{\prime} f}{\Phi f^{\prime}},
$$

${ }^{1} \Phi^{\prime \prime} t<\Phi^{\prime}$ for all $t$ implies that

$$
\int_{0}^{t}\left(\Phi^{\prime \prime} \tau-\Phi^{\prime}\right) d \tau=\left.\Phi^{\prime} \tau\right|_{0} ^{t}-2 \int_{0}^{t} \Phi^{\prime} d \tau=\Phi^{\prime} t-2 \Phi<0 .
$$

From (11), $\frac{A T C}{D L R}=\frac{\int_{0}^{t^{*}} \frac{\mathrm{I}}{T(t)}\left[f(t) \Phi^{\prime}(t)\right] d t}{\int_{0}^{t^{*}} \frac{\mathrm{I}}{T(t)}\left[f^{\prime}(t) \Phi(t)\right] d t}<\frac{\int_{0}^{t^{*}} \frac{1}{T(t)} \frac{2 f(t) \Phi(t)}{t} d t}{\int_{0}^{t^{*}} \frac{1}{T(t)}\left[f^{\prime}(t) \Phi(t)\right] d t}$

The rightmost term is less than or equal to $(2 / \gamma)$ when $\left(f^{\prime} t / f\right) \geqslant \gamma$ for all $t$. 
which states that the elasticity of the transport cost shape of the city with respect to transport cost distance equals the elasticity of the shape of the city with respect to travelling distance divided by the elasticity of transport costs with respect to travelling distance. Using (12), one may rewrite Theorem I as

Theorem $1^{\prime}:$ If $\frac{\Omega^{\prime} f}{\Omega} \gtreqless \delta$ (a constant) for all $f$, then $A T C \gtreqless \delta D L R$.

This reparameterisation is useful in many applications. Consider, for instance, a more realistic city with a Löschian hierarchy of subcentres in which all individuals shop for the same bundle of goods and purchase a particular good at the most accessible subcentre where it is available. One may then calculate, for each location, annual travel costs, and hence the residential land area for which annual travel costs are less than or equal to $f$. Since one can calculate $\Omega(f)$, this city with many subcentres has the same transport cost shape as a city with a single subcentre, unit transport costs per unit distance, and shape $\Omega(t)$. Thus, our analysis can be applied to cities with many subcentres and a complex pattern of congestion. ${ }^{1}$

\section{BENEFITS AND AGGREGATE LAND RENTS}

It is important not to confuse the relationship between aggregate transport costs and aggregate land rents, and the relationship between transport costs per unit distance and aggregate land rents. A costless transport improvement will decrease transport costs per unit distance, but, depending on the magnitude of the induced increase in lot sizes, may cause aggregate transport costs to increase, remain the same, or fall. Since the ratio of aggregate transport costs to differential land rents may be unaffected by the transport improvement, then it is quite possible for a transport improvement to result in no change or a fall in aggregate land rents. The effect of a transport improvement on aggregate land rents will depend critically on tastes, in particular the elasticity of substitution between land and other goods in consumption. Let us consider the case of a circular city with radial transport costs linearly proportional to distance and costless circumferential transportation, in which city residents have identical Cobb-Douglas utility

1 In this footnote we present another application of the transport cost shape of the city. Consider a circular city in which travel to any location from the centre of the city requires travelling first along the single radial road, and then along a circumferential road (which is a constant crow-line distance from the city centre). Travel along the radial road is costless, and transport costs are equal to circumferential distance from the radial road. The transport cost shape of this city is shown below to be

$$
\Omega(f)=4\left(r f-\frac{f^{2}}{\pi}\right), f \leqslant \frac{\pi r}{2},
$$

where $r$ is the radius of the city. Since $f \Omega^{\prime}<\Omega$ for all $f$, then from Theorem $\mathrm{I}^{\prime}, D L R>A T C$.

(i) is derived as follows. Divide the city into four symmetric quadrants, each with the radial road as its base. Consider one of these. Travel costs to all locations subtended by an angle $\theta$ of less than or equal to $f / r$ radians should be included in $\Omega(f)$. This area equals $f r / 2$. For $\pi / 2 \geqslant \theta \geqslant f / r$, transport costs exceed $f$ for $r>f / \theta$. Thus, we should integrate $r$ only from o to $f / \theta$ for $\pi / 2 \geqslant \theta \geqslant f / r$. The value of this area is $f r / 2-f^{2} / \pi$. Hence, $\Omega(f)=4\left(r f-f^{2} / \pi\right)$. 
functions and in which the opportunity rent on land is zero. ${ }^{1}$ We assume too that the transport improvement results in an equi-proportional reduction in travel costs at all locations.

It is characteristic of the Cobb-Douglas utility function that the ratio of each individual's expenditure on land to his expenditure on the private good is constant. It follows, since individuals have identical tastes, that aggregate expenditure on the private good is a constant proportion of aggregate land rents. We also know from Theorem I that for such a city, when the opportunity rent on land in non-urban use is zero, aggregate transport costs equal twice aggregate land rents. Thus, aggregate expenditure on the private good is a constant proportion of aggregate land rents. Aggregate income from production, we assume, is unaffected by the transport improvement. Putting these results together, we have that aggregate land rents, aggregate transport costs, and aggregate expenditure on other goods are unaffected by the transport improvement. Thus, reduction in unit transport costs leads to more land consumption and the same aggregate expenditure on transport costs and land rents. ${ }^{2}$

Since aggregate land rents may, in general, rise, remain the same, or fall in response to a transport improvement, the induced change in aggregate land rents by itself is, except under conditions very unlikely to be satisfied, an incorrect measure of benefits from the transport improvement. The change in aggregate land rents from a transport improvement is, however, relevant in evaluating the improvement if one is concerned with the welfare of renters vis-à-vis landowners.

\section{EXTENSIONS}

\section{IV.r. Differences between individuals}

The analysis of section II extends straightforwardly to economies with heterogeneous residents. Let $f\left(t^{\prime} ; t\right)$ be the travel costs of the individual who lives at $t$ of travelling from the city centre to $t^{\prime}$, and $T\left(t^{\prime} ; t\right)$ be the lot size that would be demanded by the individual who lives at $t$ in equilibrium if he were forced to live at $t^{\prime}$. Utility maximisation requires that

$$
R^{\prime}(t)=-\frac{f_{1}(t ; t)}{T(t ; t)},
$$

where $f_{1}(t ; t)=\left[\frac{\partial f\left(t^{\prime} ; t\right)}{\partial t^{\prime}}\right]_{t} \cdot(8)$ still holds. Substitution of $(\mathrm{r} 3)$ into $(8)$ gives

$$
D L R=\int_{0}^{t^{*}} \frac{\mathrm{I}}{T(t ; t)}\left[f_{1}(t ; t) \Phi(t)\right] d t,
$$

1 To circumvent the conceptual problems associated with the boundary resident consuming an infinite amount of land because of its zero rent, we may assume instead that the opportunity rent on land is positive but arbitrarily small.

${ }^{2}$ A transport tax has the same effects as a negative transport improvement, when the disposition of revenue is ignored. Thus, in this economy, the incidence of a transport cost tax is entirely on consumers. Aggregate land rents remain the same; some landowners near the city centre benefit, but their gains are precisely offset by losses to landowners further out. 
and

$$
\frac{A T C}{D L R}=\frac{\int_{0}^{t^{*}} \frac{\mathrm{I}}{T(t ; t)}\left[f(t ; t) \Phi^{\prime}(t)\right] d t}{\int_{0}^{t^{*}} \frac{\mathrm{I}}{T(t ; t)}\left[f_{1}(t ; t) \Phi(t)\right] d t}
$$

Equation $\left(\mathrm{II}^{\prime}\right)$ indicates that Theorem I and its Corollaries carry through with the modifications that $f(t)$ is replaced by $f(t ; t)$ and $f^{\prime}(t)$ by $f_{1}(t ; t)$.

Let $\tilde{f}(t)$ be the equilibrium transport cost function, which gives for each $t$ the transport expenditures of the individual who locates at $t$ in equilibrium. Equation $\left(\mathrm{II}^{\prime}\right)$ shows that what is relevant to the determination of $A T C / D L R$ is the elasticity of each individual's transport cost function at his equilibrium location $\left[f_{1}(t ; t) t\right] /[f(t ; t)]$ and not the elasticity of the equilibrium transport cost function $\left[\tilde{f}^{\prime}(t) t\right] /[\tilde{f}(t)]$.

To clarify the point, imagine the following scenario. The city is circular and each individual's transport cost function is linear in distance, with costs per mile depending on income. Tastes are such that, in equilibrium, the rich live closer to downtown. And the distribution of income is such that the transport expenditures incurred by the equilibrium resident at each location are equal. ${ }^{1}$ Thus, $\left[f_{1}(t ; t) t\right] /[f(t ; t)]=\mathrm{I}$ while $\left[\tilde{f}^{\prime}(t) t\right] /[\tilde{f}(t)]=0$. Application of $\left(\mathrm{II}^{\prime}\right)$ indicates that in this city $A T C=2 D L R$ since each individual's transport costs are linear in distance even though equilibrium transport costs are invariant with location. More generally, in a circular city, as long as all individuals have linear transport costs, even though they may differ in tastes, incomes, and transport costs, it is still true that aggregate transport costs are precisely twice differential land rents.

\section{IV.2. Time and money costs of travel, etc.}

It is easy to show that the results of section II generalise to the case where trip frequency is variable, where $f(t)$ is interpreted as costs per trip. ${ }^{2}$ They also generalise straightforwardly to the situation where there are both time and money costs of travel. Suppose that to travel to $t$ requires not only an expenditure of money, $f(t)$, but also an expenditure of time, $g(t)$. Time not spent travelling goes towards leisure $L$ or work $W$, which yields income $Y(W)$. The resident's maximisation problem is therefore

$$
\max _{(C, T, L, W, t)} U(C, T, L, W) \text { s.t. } \begin{cases}(\mathrm{i}) & Y(W)=C+R(t) T+f(t), \\ (\text { ii) } & \mathrm{I}=L+g(t)+W .\end{cases}
$$

${ }^{1}$ For this to be possible, residential settlement must start at some distance from the city centre.

${ }^{2}$ Let $f(t)$ be the cost per trip at $t$, and $n(t)$ be the trip frequency chosen by the person at $t$ (who maximises $U(C, T, n)$ s.t. $Y=C+R(t) T+n f(t))$. The first-order condition of the resident's maximisation problem with respect to $t$ is $-n f^{\prime}=R^{\prime} T$, and the counterpart to (II) is

$$
\frac{A T C}{D L R}=\frac{\int_{0}^{t^{*}} \frac{n(t)}{T(t)}\left[f(t) \Phi^{\prime}(t)\right] d t}{\int_{0}^{t^{*}} \frac{n(t)}{T(t)}\left[f^{\prime}(t) \Phi(t)\right] d t} .
$$

Thus, the results of Section II still hold with variable trip frequency if $f(t)$ is interpreted as costs per trip rather than overall transport costs. 
If $\lambda$ and $\theta$ are the shadow prices on the two constraints respectively, then the equation corresponding to $(7)$ is

$$
-R^{\prime}(t)=\frac{\mathrm{I}}{T(t)}\left[f^{\prime}(t)+\frac{\theta(t)}{\lambda(t)} g^{\prime}(t)\right] .
$$

$[\theta(t) / \lambda(t)]$ is the shadow value of time at $t$, which varies over locations. Proceeding as before, one obtains that the equation corresponding to (II) is

$$
\frac{A T C}{D L R}=\frac{\int_{0}^{t^{*}}[\mathrm{I} / T(t)]\left(\{f(t)+[\theta(t) / \lambda(t)] g(t)\} \Phi^{\prime}(t)\right) d t}{\int_{0}^{t^{*}}[\mathrm{I} / T(t)]\left(\left\{f^{\prime}(t)+[\theta(t) / \lambda(t)] g^{\prime}(t)\right\} \Phi(t)\right) d t} .
$$

It follows immediately from ( ${ }_{15}$ ) that Theorem I and its Corollaries go through with only minor modifications. For instance, one obtains that:

$$
\text { Where } \delta \text { is a constant, if both } \frac{f \Phi^{\prime}}{f^{\prime} \Phi} \gtreqless \delta \text { and } \frac{g \Phi^{\prime}}{g^{\prime} \Phi} \gtreqless \delta \text { for all } t \text {, }
$$

then $A T C \gtreqless \delta D L R$.

Hence, if travel time and money expenditure on transport are both linear in distance and if the city is circular, then it is still the case that ${ }_{2} D L R=A T C$. This is true even if total monetised transport costs, $f(t)+[\theta(t) / \lambda(t)] g(t)$, are not linear in distance.

A possible objection to our analysis thus far is that we have ignored housing and its durability. The analysis does in fact generalise to cities with housing. In the long run, land and structure rent may be separated and the presence of structures on the land does not affect our propositions. In the short run, land and structure rent are not separable; we refer to their sum as housing rent. In this case, the analysis must be reformulated in terms of the relationship between differential housing rents and aggregate transport costs, and our earlier theorems are applicable. ${ }^{1}$

1 We first consider the relationship between differential housing rents $(D H R)$ (which are defined analogously to differential land rents) and aggregate transport costs. We then show that when housing rent is separable into structure rent and land rent, the presence of housing on the land does not effect the propositions developed relating $A T C$ and $D L R$.

We assume that vertical transport costs are zero, and that individuals care only about the floor space of their housing, so that housing is naturally measured in units of floor space. The results generalise to more sophisticated treatments of housing.

Let $\Gamma(t)$ be the amount of housing within a distance $t$ of the city centre which we shall refer to as the housing shape of the city, $p(t)$ be rent per unit of housing, and $H(t)$ be the number of units of housing occupied by the resident at $t$. Since the individual now derives utility from housing rather than land, the individual's maximisation problem is:

$$
\max _{t, C, B} U(C, H) \text { s.t. } Y=p(t) H+f(t)+C .
$$

Proceeding as before, one obtains that the equation corresponding to ( $\mathrm{II}$ ) is

$$
\frac{A T C}{D H R}=\frac{\int_{0}^{t^{*}} \frac{\mathrm{I}}{H(t)}\left[f(t) \Gamma^{\prime}(t)\right] d t}{\int_{0}^{t^{*}} \frac{\mathrm{I}}{H(t)}\left[f^{\prime}(t) \Gamma(t)\right] d t}
$$

The results of Section II generalise. The elasticity of the shape of the city is replaced by the elasticity of the housing shape of the city, $\frac{\Gamma^{\prime}(t) t}{\Gamma(t)}$, and $D L R$ is replaced by $D H R$. continued on next page 


\section{Iv.3. Generalisation to higher dimensions}

Thus far we have assumed that urban residents are unanimous in their ranking of locations in terms of accessibility, so that location may be parameterised by a single variable, some index of accessibility. However, individuals may judge differently the relative accessibility of two locations. For instance, one may be indifferent between two commuting trips, one of which costs $\$_{1}$ and takes I5 minutes, while the other costs $\$ 0.50$ and takes 20 minutes. Another individual who values his time less may be indifferent between the trip which costs $\$$ I and takes 15 minutes, and another which costs \$o.5o and takes 30 minutes. In such cases, one needs two variables to characterise a location.

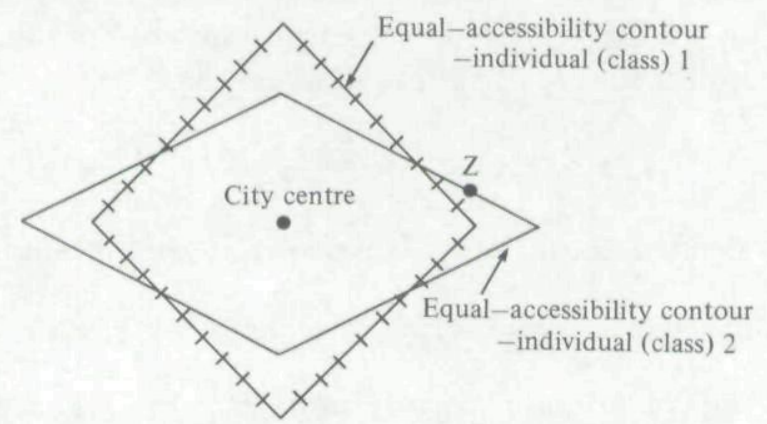

Fig. 3. Location must be indexed by two variables when individuals' equal accessibility contours do not coincide.

This can be seen from Fig. 3, which shows an equal accessibility contour for two different individuals. If one were to index locations according to individual I's travel costs, $f^{1}$, one could not write down individual 2's transport cost function, since for different locations with the same value of $f^{1}$ his transport costs would be different. Similarly, if one were to index locations according to individual 2's travel costs, one could not characterise individual I's transport costs. One can however characterise both individuals' transport cost functions using two coordinates to describe location.

In section II the analysis was considerably simplified by employing the concept of the shape of the city. This allowed us to transform any city with a complex geographical configuration into another city, equivalent for the purpose of analysis but with a simple shape. Furthermore, the shape of the city was defined in such a way that rent was the same everywhere along the boundary, as a result

cont. from page ro

When housing rent is separable into land and structure rent, the individual's maximisation problem may be written alternatively as

$$
\max _{t, \boldsymbol{H}, \boldsymbol{T}, \boldsymbol{c}} U(C, H) \text { s.t. } Y=R(t) T+\hat{p}(\mu) H+f(t)+C,
$$

where $H$ is structures, and $\hat{p}(\mu)$ is structure rent as a function of structural density $(\mu \equiv H / T)$. From this formulation, it follows that the results of Section II carry through when housing is on the land, as long as housing rent is decomposable; otherwise, differential land rents are not well-defined. 
of which there was no ambiguity in defining differential land rents. Unfortunately, this technique of simplification is not possible when residents rank locations differently in terms of accessibility. One must work instead with the actual geographical configuration of the city. In this case, land rents may not be the same everywhere along the (physical) boundaries of the city, and differential land rents may in consequence be hard to define.

We shall treat two cases in turn, first that where land rents are everywhere the same along the boundaries of the city, and then that where they are not.

\section{IV.3.I. Land rent the same everywhere along the boundaries of the city}

We shall parameterise locations using Cartesian co-ordinates, $x$ and $y$, with $(o, o)$ being the city centre (or the central business district in the case of a multinucleated city). Let $f\left(x^{\prime}, y^{\prime} ; x, y\right)$ denote the transport costs of the individual who lives at $(x, y)$ in equilibrium of travelling to $\left(x^{\prime}, y^{\prime}\right)$, and let

$$
f_{x}(x, y ; x, y) \equiv\left[\frac{\partial f\left(x^{\prime}, y^{\prime} ; x, y\right)}{\partial x^{\prime}}\right]_{(x, y)}
$$

with $f_{y}(x, y ; x, y)$ defined accordingly. It is shown in the Appendix that ${ }^{1}$

$$
D L R \gtreqless \frac{1}{2} A T C \text { if } f_{x}(x, y ; x, y) x+f_{y}(x, y ; x, y) y \gtreqless f(x, y ; x, y) \text {, for all }(x, y) \text {. }
$$

Defining $\mathbf{t}=\left[\begin{array}{ll}x & y\end{array}\right]$ (a column vector) and $\boldsymbol{\nabla} \mathbf{f}=\left[f_{x}(x, y ; x, y) f_{y}(x, y ; x, y)\right]$ (a row vector), one may write the above result more compactly as

$$
D L R \gtreqless \frac{1}{2} A T C \text { if } \boldsymbol{\nabla f ^ { \prime }} \mathbf{t} \gtreqless f \text { for all } t .
$$

$\boldsymbol{\nabla} \mathbf{f}^{\prime} \mathbf{t} / \mathbf{f}$ is the two-dimensional analog to $\left\{\left[f_{1}(t ; t) t\right] /[f(t ; t)]\right\}$.

It may at first glance appear puzzling that (I6) contains no terms reflecting the geographical configuration of the city. The reason is that when rents are the same everywhere along the physical boundaries of the city, the geographical configuration of the city is determined by the transport cost function. In illustration, we consider cities with two classes of residents, I and 2, where the transport cost function of the class $i$ is

$$
f^{i}(x, y)=a_{i} x^{\alpha}+b_{i} y^{\alpha}, \text { for } i=1,2,
$$

with $b_{1} \neq b_{2}, a_{1} \neq a_{2}$, and $\left(a_{1} / b_{1}\right) \neq\left(a_{2} / b_{2}\right)^{2}$ Furthermore, we assume that, in equilibrium, residents of both classes live at some locations along the boundary of the city. Fig. 4.i illustrates a possible geographical configuration when $\alpha=\mathrm{I}$, Fig. 4.ii one when $\alpha<\mathrm{I}$, and Fig. 4.iii one when $\alpha>\mathrm{I}$. To see how these are derived, we treat the case $\alpha=\mathrm{I}$. When $\alpha=\mathrm{I}$ each class's equal-accessibility contours are diamond-shaped, as shown in Fig. 3. Since rents are the same everywhere along the boundary, then in equilibrium all class I residents living on the boundary must be located on the same class I equal-accessibility contour,

\footnotetext{
${ }^{1}$ The mathematics employed in the Appendix is somewhat more complex than that employed in the rest of the paper. The Appendix can be skipped without loss of continuity.

${ }^{2}$ With this transport cost function, $\nabla \mathbf{f}^{\prime} \mathbf{t} \gtreqless f$ as $\alpha \gtreqless \mathrm{I}$ which implies from (16) that DLR $\frac{1}{2} A T C$ as $\alpha \gtreqless 1$.
} 
$C^{1}$; similarly, all class 2 residents living on the boundary must be located on the same class 2 equal-accessibility contour, $C^{2}$. Since land goes to the class which bids more for it, the boundary of the city is the outer envelope of $C^{1}$ and $C^{2} .^{1}$

The factor of $\frac{1}{2}$ in $(\mathrm{I} 6)$ indicates that in any city where rents are the same everywhere along its physical boundaries, the elasticity of the shape of the city (or its two-dimensional generalisation) is two. Two examples may help to clarify this remarkable result:

(a) For a city in which travel distance is crow-line distance and in which travel to all locations an equal travel distance from the city centre is equally costly, residents' equal-accessibility contours coincide and are circular. If rents are everywhere the same along the boundary of the city, the boundary coincides with an equal-accessibility contour. Thus the city is circular and $\Phi^{\prime} t / \Phi=2$.

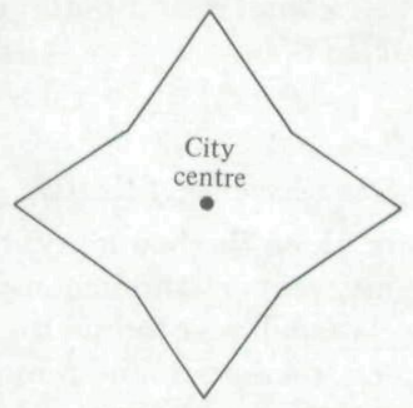

(i) Linear transport $\cos t s(\alpha=1)$

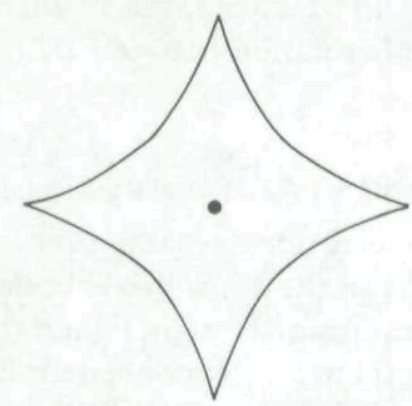

(ii) Decreasing cost to travel in both directions $(\alpha<1)$

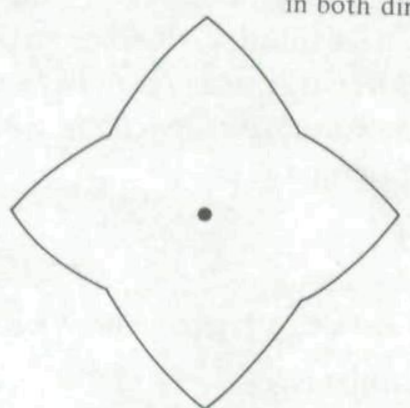

(iii) Increasing cost to travel in both directions $(\alpha>1)$

Fig. 4. Possible geographical configurations for cities when individuals rank the accessibility of locations differently: grid road systems.

(b) For a city like that in (a) except that there is a grid-road system, equalaccessibility contours and the boundary of the city are diamond-shaped. In such a city the area within travel distance $2 t$ of the city centre is four times that within

1 Suppose the equal-accessibility contours in Fig. 3 are $C^{1}$ and $C^{2}$. From the definition of $C^{1}$, an individual in group 1 is willing to bid $\bar{R}$ (the opportunity rent on land in non-urban use) per unit area for any location on $C^{1}$. Furthermore, this individual is willing to bid less than $\bar{R}$ per unit area for any location outside $C^{1}$ since it is less accessible than any location on $C^{1}$. Now consider the location $Z$ in Fig. 3. An individual from class 2 is willing to bid $\bar{R}$ per unit area for land there, since $Z$ is on $C^{2}$. An individual from class $\mathrm{I}$ is not willing to bid as much as $\bar{R}$ per unit area for land there since $Z$ is outside $C^{3}$. Thus, the land at $Z$ goes to an individual in class 2 and is on the boundary of the city since the maximum residential bid-rent there is $R$. 
travel distance $t$ and $\Phi^{\prime} t / \Phi=2$. The theorem is also evidently true for those cities shown in Fig. 4.

To recapitulate, we now consider a city with the following characteristics. It has a grid road system and an opportunity rent on land which is everywhere the same along the periphery of the city. There are $n$ groups of residents, where $n$ may be any integer number. Group $i$ 's transport costs per unit distance in the $x$-direction are $a_{i}$ and in the $y$-direction $b_{i}$. The $a_{i} \mathrm{~s}$ differ by group, as do the $b_{i} \mathrm{~s}$ and the $a_{i} / b_{i}$ s. Thus, each group ranks locations in terms of accessibility differently from every other group. This could occur if, for instance, travel in one direction were slower than in the other, and if the shadow value of time varied by group while money expenditures did not. For each group at each location $\nabla \mathbf{f}^{i} \mathbf{t}=f^{i}$. From the definition of $f$, it follows that $\nabla \mathbf{f}^{\prime} \mathbf{t}=f$, and from (16) that $D L R=\frac{1}{2} A T C$. Thus, even in this rather complex and quite realistic city, there is a simple relation between $D L R$ and $A T C$.

\section{IV.3.2. Land rent not the same everywhere along the boundaries of the city}

When land rent is not the same everywhere along the boundary, the question arises as to what is the appropriate opportunity rent on land in non-urban use to employ in computing differential land rents. We shall pose the question somewhat differently. Is there a reasonable definition of differential land rents that results in the theorems of the paper holding where (i) land rent is not the same everywhere along the physical boundary of the city, and (ii) residents do not rank locations equally in terms of accessibility? Rather strikingly, the answer is in the affirmative. If we define the opportunity rent on land at a location $(r, \theta)$, measured in polar co-ordinates, to be land rent at the boundary of the city in the direction $\theta$, then $(16)$ remains valid. ${ }^{1}$ The proof is in the Appendix.

\section{CONGLUDING REMARKS}

This paper had two central objectives:

The first was negative, to show that the commonly employed practice of inferring the benefits from a transport improvement from the changes in land rents induced by that improvement is generally not correct and may be seriously misleading. In one special case we examined, for instance, aggregate land rents are unaffected by a proportional change in transport costs to all locations.

The second was positive, to show that there are nevertheless some remarkably simple relationships between differential land rents and aggregate transport costs. For instance, in a circular city with linear transport costs, the former is exactly one-half the latter. Moreover, these relationships hold with remarkable generality; individuals can differ in tastes, incomes and transport cost functions, and the results are still valid.

Aggregate land rents and aggregate transport costs are only two of the

1 The definition can be modified to treat cities with holes in them. 
important urban economic aggregates. In a companion to this paper (1979) we show that there is also a simple relationship between aggregate land rents and expenditure on public goods. ${ }^{1}$

Queen's University, Canada

RIGHARD J. ARNOTT

Princeton University, U.S.A.

JOSEPH E. STIGLITZ

Date of receipt of final typescript: November 1980

\section{APPENDIX ${ }^{2}$}

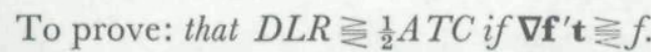

The proof is facilitated by using polar co-ordinates, in which $r$ denotes the crow-line distance from the city centre and $\theta$ the angle from the city centre in radians. To simplify the analysis we assume that there are no 'holes' in the city, namely at all locations in the city, a straight line joining the location to the city centre passes across only residential land. The proof can be extended to cities with holes.

Aggregate land rents are

$$
A L R=\int_{0}^{2 \pi}\left[\int_{0}^{\tilde{r}(\theta)} R(r, \theta) r d r\right] d \theta
$$

where $R(r, \theta)$ is land rent at $(r, \theta)$, and $\bar{r}(\theta)$ is the distance to the boundary in the direction $\theta$.

Integration of the term in square brackets in $(\mathrm{Ai})$ by parts gives

where

$$
\begin{aligned}
A L R & =\int_{0}^{2 \pi}\left\{\left[R(r, \theta) \frac{r^{2}}{2}\right]_{0}^{\bar{r}(\theta)}-\int_{0}^{\bar{r}(\theta)} R_{r}(r, \theta) \frac{r^{2}}{2} d r\right\} d \theta \\
& =\int_{0}^{2 \pi} R[\bar{r}(\theta), \theta] \frac{\bar{r}(\theta)^{2}}{2} d \theta-\int_{0}^{2 \pi} \int_{0}^{\bar{r}(\theta)} R_{r}(r, \theta) \frac{r^{2}}{2} d r d \theta
\end{aligned}
$$

$$
R_{r}(r, \theta)=\frac{\partial R(r, \theta)}{\partial r} .
$$

When land rent is the same everywhere along the boundary, $\bar{R}$, the first term on the right-hand side of (Aii) is this rent times the area of the city. When land rent is not the same everywhere along the boundary, and if we define the opportunity rent of land at $(r, \theta)$ to be the land rent at the boundary of the city in the direction $\theta$, then the first term on the right-hand side of (Aii) is the

1 There are a number of interesting extensions to the analysis. First, the theorems should extend to cities with industrial, commercial, etc. as well as residential urban land. Second, it would be useful to investigate how the relationship between aggregate transport costs and differential land rents is affected when locations differ not only in their accessibility, but also in their Ricardian characteristics such as soil fertility and quality of the microclimate. Third, one would like to know whether the actual ratio of differential land rents to aggregate transport costs is close to that indicated by the theorems presented in the paper.

${ }^{2}$ We would like to thank Jim Mirrlees for assistance in deriving this generalisation. 
aggregate opportunity rent on land in the city. In both cases, the second term on the right-hand side of (Aii) is, by definition, differential land rents; i.e.

$$
D L R=-\int_{0}^{2 \pi} \int_{0}^{\bar{r}(\theta)} R_{r}(r, \theta) \frac{r^{2}}{2} d r d \theta .
$$

Define $T\left(r^{\prime}, \theta^{\prime} ; r, \theta\right)$ to be the lot size that the individual located at $(r, \theta)$ would choose if he were forced to live at $\left(r^{\prime}, \theta^{\prime}\right)$, and $f\left(r^{\prime}, \theta^{\prime} ; r, \theta\right)$ to be the analogously defined transport cost function for the individual located at $(r, \theta)$ in equilibrium. From residents' utility-maximisation problems, with location characterised in polar co-ordinates, one obtains

where

$$
\hat{f}_{r}(r, \theta ; r, \theta)+R_{r}(r, \theta) T(r, \theta ; r, \theta)=0,
$$

$$
\hat{f}_{r}()=\left[\frac{\partial \hat{f}\left(r^{\prime}, \theta^{\prime} ; r, \theta\right)}{\partial r^{\prime}}\right]_{(r, \theta)} .
$$

Substitution of (A iv) into (Aiii) gives

$$
D L R=\int_{0}^{2 \pi} \int_{0}^{\pi(\theta)} \frac{\hat{f}_{r}(r, \theta ; r, \theta)}{T(r, \theta ; r, \theta)} \frac{r^{2}}{2} d r d \theta .
$$

Aggregate transport costs in polar co-ordinates are

$$
A T C=\int_{0}^{2 \pi} \int_{0}^{\tilde{r}(\theta)} \frac{\hat{f}(r, \theta ; r, \theta)}{T(r, \theta ; r, \theta)} r d r d \theta .
$$

Comparison of (Av) and (Avi) gives

$$
D L R \gtreqless \frac{1}{2} A T C \text { if } \hat{f}_{r} r \gtreqless \hat{f} \text { for all }(r, \theta) .
$$

One transforms from polar to Cartesian co-ordinates by using the relationships $x=r \cos \theta$ and $y=r \sin \theta$. Thus, where $f\left(x^{\prime}, y^{\prime} ; x, y\right)$ is an individual's transport cost function in Cartesian co-ordinates,

Also,

$$
f\left(x^{\prime}, y^{\prime} ; x, y\right)=f\left(r^{\prime} \cos \theta^{\prime}, r^{\prime} \sin \theta^{\prime} ; r \cos \theta, r \sin \theta\right) .
$$

$$
\begin{aligned}
f_{r}(x, y ; x, y) & \equiv\left[\partial f\left(r^{\prime} \cos \theta^{\prime}, r^{\prime} \sin \theta^{\prime} ; r \cos \theta, r \sin \theta\right) / \partial r^{\prime}\right]_{(r, \theta)} \\
& =f_{x}(x, y ; x, y) \cos \theta+f_{y}(x, y ; x, y) \sin \theta,
\end{aligned}
$$

where $f_{x}(x, y ; x, y) \equiv\left[\partial f\left(x^{\prime}, y^{\prime} ; x, y\right) / \partial x^{\prime}\right]_{(x, y)}$ and $f_{y}$ is defined accordingly. Using $x=r \cos \theta$ and $y=r \sin \theta$ again, one may rewrite (Aviii) as

$$
f_{r}=\frac{\mathrm{I}}{r}\left[f_{x}(x, y ; x, y) x+f_{y}(x, y ; x, y) y\right],
$$

or

$$
f_{r} r=f_{x} x+f_{y} y
$$

Now, $\hat{f}(x, y ; x, y)=f(r, \theta ; r, \theta)$, and $f_{r}(x, y ; x, y)=\hat{f}_{r}(r, \theta ; r, \theta)$. Thus, $f_{r} r=\hat{f}_{r} r$. Too, $f_{x} x+f_{y} y \equiv \boldsymbol{\nabla} \mathbf{f}^{\prime} \mathbf{t}$ where $\boldsymbol{\nabla f}=\left[\begin{array}{ll}f_{x} & f_{y}\end{array}\right]$ and $\mathbf{t}=\left[\begin{array}{ll}x & y\end{array}\right]$. Combining these results, (Aix), and (Avii) gives

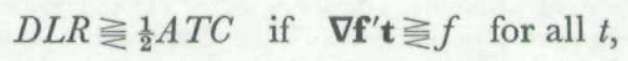

which is the result presented in the text. 
Where land rent is not the same everywhere along the boundary of the city with other reasonable definitions of differential land rents, (i6) does not obtain. For instance, if the opportunity rent on land in non-urban use is defined as the minimum rent on land at the boundary of the city, $\tilde{R}$, then, where $\overline{D L R}$ denotes differential land rents according to this alternative definition,

$$
\begin{aligned}
\overline{D L R} & =\int_{0}^{2 \pi}\{R[\bar{r}(\theta), \theta]-\tilde{R}\} \frac{\bar{r}(\theta)^{2}}{2} d \theta-\int_{0}^{2 \pi} \int_{0}^{\bar{r}(\theta)} R_{r}(r, \theta) \frac{r^{2}}{2} d r d \theta \\
& =\int_{0}^{2 \pi}\{R[\bar{r}(\theta), \theta]-\tilde{R}\} \frac{\bar{r}(\theta)^{2}}{2} d \theta+\int_{0}^{2 \pi} \int_{0}^{\bar{r}(\theta)} \frac{\hat{f}_{r}(r, \theta ; r, \theta)}{T(r, \theta ; r, \theta)} \frac{r^{2}}{2} d r d \theta,
\end{aligned}
$$

(Avi) still applies. Thus, the relationship between $A T C$ and $\overline{D L R}$ is not as simple as that between $A T C$ and $D L R$.

The central results derived in this Appendix and in the main body of the paper are essentially geometric. There are analogous results for higher dimensions. We do not present them since their economic importance is not apparent.

\section{References}

Arnott, R. J. (1975). 'Topics in Residential Location Theory'. Unpublished Ph.D. thesis, Yale University.

and Stiglitz, J. E. (1979). 'Aggregate land rents, expenditure on public goods, and optimal city size'. Quarterly Journal of Economics (November), Vol. 93, no. 4, pp. 471-500.

Bollobas, B. and Stern, N. (1972). 'The optimum structure of market areas'. Journal of Economic Theory, vol. 4 , no. 2 (April), pp. 174-9.

Fishlow, A. (1965). Railroads and the Transformation of the Ante-Bellum Economy. Cambridge: Harvard University Press.

Fogel, R. W. (1964). Railroads and American Economic Growth: Essays in Economic History. Baltimore: John Hopkins Press.

Getz, M. (1975). 'A model of the impact of transportation investments on land rents'. Journal of Public Economics, vol. 4, no. I (February), pp. 57-74.

Mohring, H. (196r). 'Land values and the measurement of highway benefits'. Journal of Political Economy, vol. 69, no. 3 (June), pp. 236-49.

Polinsky, A. M. and Shavell, S. (1976). 'Amenities and property values in a model of an urban area'. Journal of Public Economics, vol. 5, no. 1, 2 January-February, pp. 119-29. 
Copyright of Economic Journal is the property of Blackwell Publishing Limited. The copyright in an individual article may be maintained by the author in certain cases. Content may not be copied or emailed to multiple sites or posted to a listserv without the copyright holder's express written permission. However, users may print, download, or email articles for individual use. 